\title{
The association of serum uromodulin with allograft function and risk of urinary tract infection in kidney transplant recipients
}

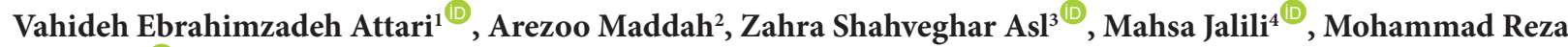 \\ Ardalan $^{2 *}$, Saman Mokari ${ }^{2}$ \\ ${ }^{1}$ Maragheh University of Medical Sciences, Maragheh, Iran \\ ${ }^{2}$ Kidney Research Center, Tabriz University of Medical Sciences, Tabriz, Iran \\ ${ }^{3}$ Nutrition Research Center, Tabriz University of Medical Sciences, Tabriz, Iran \\ ${ }^{4}$ Cell, Molecular Biology and Genomics Group, Department of Biology, Norwegian University of Science and Technology, Trondheim, Norway
}

\section{A R T I C L E I N F O}

Article Type:

Original

\section{Article History:}

Received: 27 October 2019

Accepted: 10 January 2020

Published online: 3 February 2020

\section{Keywords:}

Uromodulin, Urinary tract infections, Kidney function, Kidney transplantation, Chronic kidney disease, Glomerular filtration rate, Endstage renal disease

\begin{abstract}
A B S T R A C T
Introduction: There is some evidence to suggest that low levels of uromodulin in urine and serum are associated with decreased renal function and increased mortality risk. Objectives: This study is designed to measure circulating uromodulin levels and explore their relationship to urinary tract infection and renal function in kidney transplant recipients. Patients and Methods: In this cross-sectional study, 90 eligible kidney transplant recipients were evaluated 6-12 months of post-transplantation. Fasting blood samples were taken to determine the serum level of uromodulin with urea, creatinine, and other biochemical characteristics. Urine samples were taken for analysis and culture. Kidney function was estimated based on the chronic kidney disease epidemiology collaboration (CKD-EPI) creatinine equation and the modification of diet in renal disease (MDRD) equation.

Results: Patients' serum uromodulin levels were significantly correlated with their serum creatinine $(P=0.024)$ and estimated glomerular filtration rate by the EPI equation (eGFREPI, $P=0.038)$. There was no significant association between serum uromodulin levels and incidence of urinary tract infection of post-transplantation $(P>0.05)$.

Conclusion: Serum and urine uromodulin levels may be regarded as the predictive indicators of renal function. However, given the lack of studies on the association between serum uromodulin levels and urinary tract infection risks in kidney transplant recipients, further research is needed to clarify uromodulin's protective effect against urinary tract infection.
\end{abstract}

Implication for health policy/practice/research/medical education:

In a study on 90 eligible kidney transplant recipients, we found serum uromodulin levels are significantly related to serum creatinine levels and estimated glomerular filtration rate, but there is no significant association between serum uromodulin levels and urinary tract infection incidence.

Please cite this paper as: Ebrahimzadeh Attari V, Maddah A, Shahveghar Asl Z, Jalili M, Ardalan ME, Mokari S. The association of serum uromodulin with allograft function and risk of urinary tract infection in kidney transplant recipients. J Renal Inj Prev. 2021; 10(1): e02. doi: 10.34172/jrip.2021.02.

\section{Introduction}

Uromodulin, also known as the Tamm-Horsfall protein, is a glycoprotein produced by the thick ascending limb of the loop of Henle and the early distal convoluted tubule $(1,2)$. It is the most abundantly excreted protein in the urine of healthy adults and plays several important roles, including the prevention of renal stones, the regulation of arterial blood pressure, and the prevention of urinary tract infection by binding and inhibiting bacterial adhesion to the uroepithelium (3-9). Uromodulin is also present in serum, but its molecular structure and functions are not well-known (3).

Based on the results of epidemiological studies, urinary uromodulin appears to have a direct correlation with kidney size and renal function as measured by glomerular filtration rate (GFR), and also has a reverse association with increasing the age (over 60 years) and diabetes $(1,10)$. In old age, high serum uromodulin levels are accompanied 
by a slow decrease in the GFR and a reduction in the risk of urinary tract infection and mortality $(1,11,12)$.

Despite the recent increase in survival rates, the longterm outcomes of kidney transplant have not significantly improved. Urinary tract infection affects about 5\%-36\% of kidney transplant recipients, while both are the most prevalent infectious disease in this group and may lead to reduced kidney function $(13,14)$.

Both serum and urine uromodulin may protect kidney against urinary infections, and they may be considered to be as the biomarkers of renal function $(1-3,15,16)$. The measurement of serum uromodulin levels is easier than that of urinary uromodulin levels, since the latter requires 24-hour urinary collection (1). Therefore, measuring serum uromodulin levels may serve to assess the proportion of healthy nephrons in patients with chronic kidney disease (CKD) (17). However, it is still not entirely clear whether uromodulin levels can predict urinary tract infection.

\section{Objectives}

Studies on the association between serum uromodulin levels and renal function in kidney transplant recipients are limited. Moreover, uromodulin's relationship with urinary tract infection has not yet been investigated. The present study was conducted to evaluate serum uromodulin level and its association with urinary tract infection and kidney function in kidney transplant recipients.

\section{Patients and Methods}

Study design and subjects

A cross-sectional study was conducted on kidney transplant recipients referred to the Imam Reza hospital (Tabriz, Iran). Inclusion criteria were age 18-70 years and appropriate kidney function, as indicated by serum creatinine levels $<1.6 \mathrm{mg} / \mathrm{dL}$. Exclusion criteria were incidence of acute kidney rejection during the first months after kidney transplant recipients, incidence of serious infections (e.g., HIV, CMV, and HBV), active or chronic infection or inflammatory disorders, and concurrent treatment with antibiotics. A total of 90 patients were enrolled voluntarily 6-12 months after kidney transplant recipients, and they signed a written consent form prior to the intervention.

\section{Anthropometric and biochemical assessments}

General information on age, gender, transplantation type, education, and smoking habits was collected from participants at baseline. Subjects' height without shoes was measured using a standard tape with $0.1 \mathrm{~cm}$ precision. Their body weight (including light clothing and excluding shoes) was determined on a calibrated Seca scale with $0.1 \mathrm{~kg}$ precision (Seca 762; Vogel and Halke, Hamburg, Germany). Their body mass index (BMI) [weight (kg)/ height $(\mathrm{m})^{2}$ ] was calculated, as was their weight one month after transplantation. Blood pressure was measured with a mercury sphygmomanometer placed around each subject's right arm while there were in a seated position after a 5-minute rest period.

Venous blood samples were drawn after an overnight fast. Fasting blood sugar, serum urea, creatinine, cholesterol, and triglyceride levels were measured through standard clinical laboratory methods. Serum uromodulin levels were measured using the human ELISA kit (Euroimmun Medizinische Labordiagnostika AG, Lübeck, Germany), according to the manufacturer's instructions. GFR was considered to be the best overall index of kidney function and estimated based on the chronic kidney disease epidemiology collaboration (CKDEPI) creatinine equation (2009) and the modification of diet in renal disease (MDRD) equation, using the online eGFR calculator on the national kidney foundation website (https://www.kidney.org/professionals/KDOQI/ gfr_calculator). A single urine sample was taken from each patient for analysis and culture. Urine sampling method was trained to minimize false positive results.

\section{Ethical issues}

Human rights were respected in accordance with the Helsinki Declaration 1975, as revised in 1983. The ethical committee of Tabriz University of Medical Sciences approved this study (ethical code; IR.TBZMED. REC.1396.585). The informed consents were taken from the patients. Besides, this study was extracted from the M.D thesis of Saman Mokari, (the project number of 58351) at this University.

\section{Statistical analysis}

Data were analyzed using SPSS software, version 21.0 (IBM Corp., Armonk, NY, USA). The normal distribution of variables was evaluated through the KolmogorovSmirnov test. Results were reported as means (SD) if not otherwise stated. For the association between serum uromodulin levels and kidney function was assessed through Pearson's correlation test since and the linear regression test (after adjusting for age and gender). In addition, the association between serum uromodulin levels with the incidence of urinary tract infection was determined using Spearman's rho correlation test and a logistic regression test after adjusting for age and gender, since the significance level was set at $P=0.05$.

\section{Results}

Adjusted to inclusion criteria, this study consisted of 90 kidney transplant recipients. Participants' general and demographic characteristics are presented in Table 1. Their mean age was $44.1 \pm 13.05$ years, since $41.1 \%$ of them were women. Their anthropometric and serum biochemical characteristics are presented in Table 2. 
Their average body weight and BMI were $69.61 \pm 11.59 \mathrm{~kg}$ and $25.77 \pm 4.42 \mathrm{~kg} / \mathrm{m}^{2}$, respectively. Mean body weight change 6-12 months after transplantation (average 7.46 \pm 1.65 months) was $4.06 \pm 7.45 \mathrm{~kg}$.

The mean serum levels of urea and creatinine were $38.78 \pm 12.19 \mathrm{mg} / \mathrm{dL}$ and $1.26 \pm 0.25 \mathrm{mg} / \mathrm{dL}$, respectively. The GFR was $63.62 \pm 15.02 \mathrm{~mL} / \mathrm{min}$ according to the CKD-EPI equation and $57.45 \pm 12.61 \mathrm{~mL} / \mathrm{min}$ according to the MDRD equation. Average serum uromodulin levels were $85.35 \pm 44.34 \mathrm{ng} / \mathrm{mL}$. Around, 68 out of 90 kidney transplant recipients, (75.6\%) had negative urine culture, while $14(15.6 \%)$ patients had positive urine culture indicating the existence of urinary tract infection.

Table 1. The demographic information of participants $(n=90)$

\begin{tabular}{lc}
\hline & No. (\%) \\
\hline Age* & $44.1 \pm 13.05$ \\
Gender & $37(44.1)$ \\
$\quad$ Women & $53(58.9)$ \\
$\quad$ Men & \\
Transplant type & $76(84.4)$ \\
Living donor & $14(15.6)$ \\
Dead brain donor & \\
Smoking & $80(88.9)$ \\
Yes & $10(11.1)$ \\
No & \\
Literacy & $19(21.1)$ \\
Illiterate & $37(41.1)$ \\
Under diploma & $22(24.4)$ \\
Diploma & $3(3.3)$ \\
Undergraduate degree & $9(10)$ \\
Bachelor and higher & \\
\hline
\end{tabular}

* Mean \pm SD.

Table 2. The anthropometric and serum biochemical characteristics of participants $(n=90)$

\begin{tabular}{lc}
\hline & Mean \pm SD \\
\hline Weight $(\mathrm{kg})$ & $69.61 \pm 11.59$ \\
Weight differences after transplantation & $4.06 \pm 7.45$ \\
BMI $\left(\mathrm{kg} / \mathrm{m}^{2}\right)$ & $25.77 \pm 4.42$ \\
SBP $(\mathrm{mm} \mathrm{Hg})$ & $122.93 \pm 11.39$ \\
DBP $(\mathrm{mm} \mathrm{Hg})$ & $76.41 \pm 9.36$ \\
FBS $(\mathrm{mg} / \mathrm{dL})$ & $108.26 \pm 34.65$ \\
Total cholesterol $(\mathrm{mg} / \mathrm{dL})$ & $187.48 \pm 43.6$ \\
Triglyceride $(\mathrm{mg} / \mathrm{dL})$ & $189.39 \pm 114.79$ \\
Urea $(\mathrm{mg} / \mathrm{dL})$ & $38.78 \pm 12.19$ \\
Creatinine $(\mathrm{mg} / \mathrm{dL})$ & $1.26 \pm 0.25$ \\
eGFR-EPI $(\mathrm{ml} / \mathrm{min})$ & $63.62 \pm 15.02$ \\
eGFR-MDRD $(\mathrm{mL} / \mathrm{min})$ & $57.45 \pm 12.61$ \\
Uromodulin $(\mathrm{ng} / \mathrm{mL})$ & $85.35 \pm 44.34$ \\
Urine culture* & \\
Yes & $68(75.6 \%)$ \\
No & $14(15.6 \%)$ \\
\hline
\end{tabular}

* Data are presented as No. (\%).
The correlation of serum uromodulin levels with kidney function, urinary tract infection incidence, and other baseline parameters are summarized in Tables 3 and 4 . Serum uromodulin levels were significantly correlated with serum creatinine levels $(P=0.024)$ and eGFR-EPI values $(P=0.038)$ since; the correlation coefficient was approximately 0.2 in both cases, indicating weak but significant relationships. However, according to Tables 3 and 4, serum uromodulin levels had no statistically significant relationship with the incidence of urinary tract infection and BMI $(P>0.05)$.

According to Table 5, serum uromodulin levels also showed a significant association with serum creatinine levels and eGFR-EPI values based on a linear regression test after adjusting for age and gender. There was a significant and positive linear association between serum uromodulin levels and eGFR-EPI values (Figure 1). Serum uromodulin levels showed no significant association with urinary tract infection incidence based on the logistic regression test after adjusting for age and gender.

\section{Discussion}

Survival is the main goal of post-kidney transplant recipients care, therefore identifying biomarkers of kidney

Table 3. The correlation of serum Uromodulin with kidney function and urinary tract infection

\begin{tabular}{lcc}
\hline & $\boldsymbol{r}$ & $\boldsymbol{P}_{\text {value }}$ \\
\hline Serum urea $(\mathrm{mg} / \mathrm{dL})$ & -0.201 & 0.075 \\
Serum creatinine $(\mathrm{mg} / \mathrm{dL})$ & -0.254 & 0.024 \\
eGFR -EPI (mL/min) & 0.234 & 0.038 \\
eGFR - MDRD (mL/min) & 0.220 & 0.052 \\
UTI $^{\text {b }}$ & -0.032 & 0.789 \\
\hline
\end{tabular}

MDRD, modification of diet in renal disease; UTI, urinary tract infection; eGFR, estimated glomerular filtration rate.

a $P$ values are based on the Pearson correlation test.

${ }^{b} P$ value is based on the Spearman rho correlation test.

Table 4. The association of serum Uromodulin with some baseline parameters

\begin{tabular}{lll}
\hline & $\boldsymbol{r}$ & $\boldsymbol{P}$ value $^{\mathrm{a}}$ \\
\hline Gender & -0.118 & 0.302 \\
Age & -0.043 & 0.710 \\
Weight $(\mathrm{kg})$ & -0.050 & 0.664 \\
Weight differences & -0.169 & 0.138 \\
BMI $\left(\mathrm{kg} / \mathrm{m}^{2}\right)$ & -0.007 & 0.949 \\
FBS $(\mathrm{mg} / \mathrm{dL})$ & 0.029 & 0.838 \\
Total cholesterol $(\mathrm{mg} / \mathrm{dL})$ & 0.131 & 0.327 \\
Triglyceride $(\mathrm{mg} / \mathrm{dL})$ & -0.039 & 0.772 \\
SBP $(\mathrm{mm} \mathrm{Hg})$ & -0.005 & 0.963 \\
DBP $(\mathrm{mm} \mathrm{Hg})$ & -0.004 & 0.970
\end{tabular}

BMI, body mass index, FBS, fasting blood sugar; SBP, systolic blood pressure; DBP, diastolic blood pressure

a $P$ value is based on the Spearman rho correlation test. 
Table 5. The association of serum Uromodulin with kidney function and urinary tract infection

\begin{tabular}{lcc}
\hline & B $(\mathbf{9 5 \%} \mathbf{C l})$ & $\boldsymbol{P}$ value $^{\mathrm{a}}$ \\
\hline Serum urea $(\mathrm{mg} / \mathrm{dL})$ & $-0.048(-0.108,0.013)$ & 0.121 \\
Serum creatinine $(\mathrm{mg} / \mathrm{dL})$ & $-0.001(-0.002,0.000)$ & 0.040 \\
eGFR -EPI $(\mathrm{mL} / \mathrm{min})$ & $0.071(0.001,0.141)$ & 0.048 \\
eGFR - MDRD (mL/min) & $0.058(-0.003,0.119)$ & 0.061 \\
UTI $^{\text {b }}$ & $1.004(0.985,1.024)$ & 0.653 \\
\hline
\end{tabular}

UTI; urinary tract infection.

a $P$ values are based on the linear regression test after adjusting for age and sex.

${ }^{\mathrm{b}} P$ values are based on the logistic regression test after adjusting for age and gender.

function and other health issues in kidney transplant recipients is highly relevant. Uromodulin is the most abundant urinary protein in mammals and plays many important roles, including maintenance of water and electrolyte balance, ion transport, and bacterial clearance from the urinary tract. Low, urinary uromodulin and serum uromodulin levels have been associated with increased mortality risk and therefore may be useful markers of cardiovascular and renal function (18).

According to our findings, a significant and positive linear association between serum uromodulin levels and eGFR-EPI values was seen. Similarly, Steubl et al reported that serum uromodulin levels may anticipate graft function like eGFR, 1-3 months after renal transplantation, since decreased serum uromodulin levels are related to ensuing graft loss (19). It has also been previously reported that urinary uromodulin levels are higher in kidney transplant recipients than in other patients, while that they are prone to graft dysfunction and outcome 5.3 years after transplant (20). Risch et al showed that serum uromodulin level had inverse associations with serum levels of creatinine and urea, and a positive correlation with eGFR values in healthy participants (15). Additionally, Sejdiu et al found an association between low urinary uromodulin levels and higher risk of end-stage renal disease and cardiovascular disease mortality among 131 patients with type 1 diabetes (21). According to a large cohort study on communitydwelling adults in Pittsburgh, higher urinary uromodulin levels are associated with a lower incidence of CKD (22). It has also been reported that high uromodulin levels in patients with CKD may anticipate the development of the disease (23).

While uromodulin's protective role on kidney function is not fully understood, it may involve anti-inflammatory effects after tubular injury and prevention of urinary tract infection and stone formation $(1,24)$. Higher urinary uromodulin levels have been suggested to indicate improved tubular functions, including production of erythropoietin and maintenance of acid-base and mineral metabolism homeostasis (24).

The present study has not shown a significant

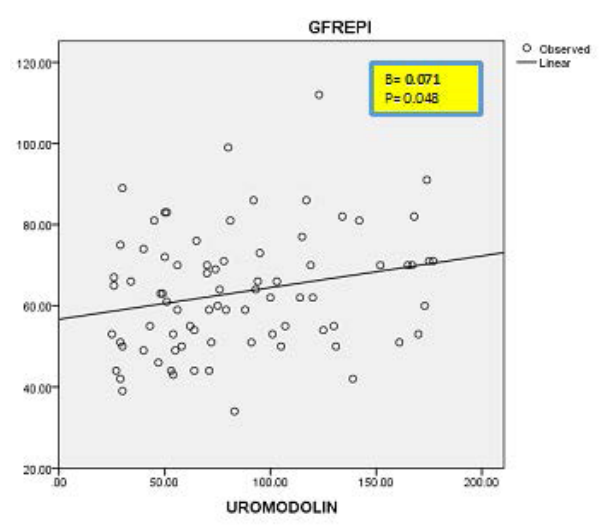

Figure 1. The linear association between serum Uromodulin and eGFR -EPI based on the linear regression model.

association between serum uromodulin levels and the incidence of urinary tract infection in kidney transplant recipients. While no prior study assessing this association, however, there is some evidence that, urinary uromodulin has a protective effect against urinary tract infection. According to the study by Stahl et al, low urinary uromodulin levels are significantly associated with a higher risk of developing urinary tract infection, higher incidence of initial graft dysfunction, and the need for dialysis in kidney transplant patients (25).

The results of a prospective longitudinal cohort study with older community-dwelling adults have shown that higher urinary uromodulin levels are significantly associated with lower urinary tract infection incidence, independent of other traditional urinary tract infection risk factors (12). Likewise, another prospective cohort study on patients undergoing coronary angiography demonstrated an association between higher serum uromodulin concentrations and a reduced risk of mortality due to fatal infections (18). Moreover, experimental studies with uromodulin knockout mice have shown increased susceptibility to urinary tract infection (26). As one of the first-line host defenses against bacterial urinary tract infection, uromodulin could bind and inhibit bacterial adhesion to the uroepithelium and facilitate the urinary washout of bacteria. On the other hand, it can induce protective immune responses against uropathogenic bacteria. However, its role in preventing urinary tract infection in humans has not been definitively proved $(12,27)$.

\section{Conclusion}

According to results, serum uromodulin levels are significantly related to serum creatinine levels and eGFR values, but there is no significant association between serum uromodulin levels and urinary tract infection incidence in kidney transplant recipients. Further studies are needed to investigate this relationship, as well as the 
association between serum uromodulin and, urinary uromodulin levels and their post- kidney transplant recipient's time-trend.

\section{Study limitations}

The main limitation of the present cross-sectional study was its small sample size. Moreover, it could have benefited from the measurement of urinary uromodulin levels in order to assess their relationship to serum uromodulin levels.

\section{Authors' contribution}

VEA contributed to design of the study, gathering the patients, data entering and data analysis and also preparing the manuscript; $\mathrm{ZSH}$ prepared the primary draft of article; MJ contributed in preparing the manuscript; MRA cooperated in design of the study and final edit of the manuscript; AM and SP contributed to gathering the patients. All authors read and signed the paper manuscript.

\section{Conflicts of interest}

The authors declared that there was no conflict of interest.

\section{Ethical considerations}

Ethical issues including plagiarism, double publication, and redundancy have been completely observed by the authors.

\section{Funding/Support}

This study was supported by a grant from the Research Vice-Chancellor and Kidney Research Center, Tabriz University of Medical Sciences, Tabriz, Iran. The results of this article are derived from MD thesis of Saman Mokari.

\section{References}

1. Garimella PS, Biggs ML, Katz R, Ix JH, Bennett MR, Devarajan $\mathrm{P}$, et al. Uromodulin in kidney health and disease. Current opinion in nephrology and hypertension. Kidney Int. 2015;88:1126-34. doi: 10.1038/ki.2015.192.

2. Devuyst O, Olinger E, Rampoldi L. Uromodulin: from physiology to rare and complex kidney disorders. Nat Rev Nephrol. 2017;13525-544. doi: 10.1038/nrneph.2017.101.

3. Scherberich JE, Gruber R, Nockher WA, Christensen EI, Schmitt H, Herbst V, et al. Serum uromodulin-a marker of kidney function and renal parenchymal integrity. Nephrol Dial Transplant. 2018;33:284-295. doi: 10.1093/ ndt/gfw422.

4. Mo L, Huang HY, Zhu XH, Shapiro E, Hasty DL, Wu XR. Tamm-Horsfall protein is a critical renal defense factor protecting against calcium oxalate crystal formation. Kidney Int. 2004;66:1159-66. doi: 10.1111/j.15231755.2004.00867.x.

5. Graham LA, Padmanabhan S, Fraser NJ, Kumar S, Bates JM, RaffiHS, etal.Validation of uromodulin as a candidategenefor human essential hypertension. Hypertension. 2014;63:551-

\section{8.doi:10.1161/HYPERTENSIONAHA.113.01423.}

6. Trudu M, Janas S, Lanzani C, Debaix H, Schaeffer C, Ikehata $\mathrm{M}$ et al. Common noncoding UMOD gene variants induce salt-sensitive hypertension and kidney damage by increasing uromodulin expression. Nat Med. 2013;19:165560. doi: $10.1038 / \mathrm{nm} .3384$.

7. Scolari F, Izzi C, Ghiggeri GM. Uromodulin: from monogenic to multifactorial diseases. Nephrol Dial Transplant. 2015;30:1250-6. doi: 10.1093/ndt/gfu300.

8. Iorember FM, Vehaskari VM. Uromodulin: old friend with new roles in health and disease. Pediatr Nephrol. 2014;29:1151-8. doi: 10.1007/s00467-013-2563-z.

9. Bostom AG, Steubl D, Friedman AN. Hypothesis: potential utility of serum and urine uromodulin measurement in kidney transplant recipients? Transplant Direct. 2017;6; 3:e219. doi: 10.1097/TXD.0000000000000737.

10. Pruijm M, Ponte B, Ackermann D, Paccaud F, Guessous I, Ehret $G$, et al. Associations of urinary uromodulin with clinical characteristics and markers of tubular function in the general population. Clin J Am Soc Nephrol. 2016;11:7080. doi: 10.2215/CJN.04230415.

11. Garimella PS, Biggs ML, Katz R, Ix JH, Bennett MR, Devarajan $\mathrm{P}$, et al. Urinary uromodulin, kidney function, and cardiovascular disease in elderly adults. Kidney Int. 2015; 88(5):1126-34. doi: 10.1038/ki.2015.192.

12. Garimella PS, Bartz TM, Ix JH, Chonchol M, Shlipak MG, Devarajan P, et al. Urinary uromodulin and risk of urinary tract infections: the cardiovascular health study. Am J Kidney Dis. 2017;69:744-51. doi: 10.1053/j. ajkd.2016.08.022.

13. Espinar MJ, Miranda IM, Costa-de-Oliveira S, Rocha R, Rodrigues AG, Pina-Vaz C. Urinary tract infections in kidney transplant patients due to escherichia coli and Klebsiella pneumoniae-producing extended-spectrum $\beta$-lactamases: risk factors and molecular epidemiology. PLoS One. 2015;10:e0134737. doi: 10.1371/journal. pone. 0134737 .

14. Mitra S, Alangaden GJ. Recurrent urinary tract infections in kidney transplant recipients. Curr Infect Dis Rep. 2011;13:579-87. doi: 10.1007/s11908-011-0210-z.

15. Risch L, Lhotta K, Meier D, Medina-Escobar P, Nydegger UE, Risch M. The serum uromodulin level is associated with kidney function. Clin Chem Lab Med. 2014;52:175561. doi: 10.1515/cclm-2014-0505.

16. Steubl D, Block M, Herbst V, Nockher WA, Schlumberger W, Satanovskij R, et al. Plasma uromodulin correlates with kidney function and identifies early stages in chronic kidney disease patients. Medicine (Baltimore). 2016;95:e3011. doi: 10.1097/MD.0000000000003011.

17. Fedak D, Kuźniewski M, Fugiel A, Wieczorek-Surdacka E, Przepiórkowska-Hoyer B, Jasik P, et al. Serum uromodulin concentrations correlate with glomerular filtration rate in patients with chronic kidney disease. Pol Arch Med Wewn. 2016;126:995-1004. doi: 10.20452/pamw.3712.

18. Delgado GE, Kleber ME, Scharnagl H, Krämer BK, März W, Scherberich JE. Serum uromodulin and mortality risk in patients undergoing coronary angiography. J Am Soc Nephrol. 2017;28:2201-10. doi: 10.1681/ASN.2016111162.

19. Steubl D, Block M, Herbst V, Schlumberger W, Nockher A, Angermann S, et al. Serum uromodulin predicts graft 
failure in renal transplant recipients. Biomarkers. 2017; 22:171-7. doi: 10.1080/1354750X.2016.1252957.

20. Reznichenko A, van Dijk MC, van der Heide JH, Bakker SJ, Seelen M, Navis G. Uromodulin in renal transplant recipients: elevated urinary levels and bimodal association with graft failure. Am J Nephrol. 2011;34:445-51. doi: $10.1159 / 000332231$.

21. Sejdiu I, Torffvit O. Decreased urinary concentration of Tamm-Horsfall protein is associated with development of renal failure and cardiovascular death within 20 years in type 1 but not in type 2 diabetic patients. Scand J Urol Nephrol. 2008;42:168-74. doi: 10.1080/00365590701644691.

22. Leiherer A, Muendlein A, Saely CH, Kinz E, Brandtner EM, Fraunberger P, et al. Serum uromodulin is associated with impaired glucose metabolism. Medicine (Baltimore). 2017;96:e5798. doi: 10.1097/MD.0000000000005798.

23. Tan F, Zeng Y, Yan L, Zhang D. Low plasma uromodulin is a predictor of early stage chronic kidney disease progression. Int J Clin Exp Med. 2017;10:8055-9.
24. Garimella PS, Katz R, Ix JH, Fried LF, Kritchevsky SB, Devarajan P, et al. Association of urinary uromodulin with kidney function decline and mortality: the health ABC study. Clin Nephrol. 2017;87:278-86. doi: 10.5414/ CN109005.

25. Stahl K, Beneke J, Haller H, Gwinner W, Schiffer M. Reduced Urinary uromodulin (UMOD)-levels sre associated with urinary tract infections (UTI) after renal transplantion. 2015 American Transplant Congress; May 3, 2015; Sunday. Abstract number: B17.

26. Bates JM, Raffi HM, Prasadan K, Mascarenhas R, Laszik Z, Maeda N, et al. Tamm-Horsfall protein knockout mice are more prone to urinary tract infection: rapid communication. Kidney Int. 2004;65:791-7. doi: 10.1111/j.1523-1755.2004.00452.x.

27. Säemann MD, Weichhart T, Hörl WH, Zlabinger GJ. Tamm- Horsfall protein: a multilayered defence molecule against urinary tract infection. Eur J Clin Invest. 2005;35:227-35. doi: 10.1111/j.1365-2362.2005.01483.x.

Copyright $\odot 2021$ The Author(s); Published by Nickan Research Institute. This is an open-access article distributed under the terms of the Creative Commons Attribution License (http://creativecommons.org/licenses/by/4.0), which permits unrestricted use, distribution, and reproduction in any medium, provided the original work is properly cited. 